\title{
Planar graphs of maximum degree six without 7-cycles are class one
}

\author{
Danjun Huang* $\quad$ Weifan Wang $^{\dagger}$ \\ Department of Mathematics \\ Zhejiang Normal University \\ Jinhua 321004, China \\ $\{$ hdanjun,wwf $\}$ ezjnu.cn
}

Submitted: Aug 10, 2010; Accepted: Jul 28, 2012; Published: Aug 9, 2012

Mathematics Subject Classifications: 05C15

\begin{abstract}
It is conjectured by Vizing (1965) that every planar graphs graph $G$ with maximum degree $6 \leqslant \Delta \leqslant 7$ is class one. The case $\Delta=7$ was confirmed independently by Sanders and Zhao (2001), and by Zhang (2000). In this paper, we prove that every planar graph $G$ with $\Delta=6$ and without 7 -cycles is class one.
\end{abstract}

Keywords: Planar graph; edge coloring; class one; cycle

\section{Introduction}

In this paper, all graphs under consideration are simple and finite. A plane graph is a particular drawing of a planar graph on the Euclidean plane. Let $V(G), E(G), F(G)$ and $\Delta(G)$ (or $\Delta$ for short) be the vertex set, edge set, face set, and maximum degree of a given plane graph $G$, respectively. Let $C_{n}$ denote a cycle of length $n$. We say that $G$ is $C_{n}$-free if $G$ contains no $C_{n}$ as a subgraph.

An edge $k$-coloring of a graph $G$ is a function $\phi: E(G) \mapsto\{1,2, \ldots, k\}$ such that any two adjacent edges receive different colors. The edge chromatic number, denoted $\chi^{\prime}(G)$, of a graph $G$ is the smallest integer $k$ such that $G$ has an edge $k$-coloring. The celebrated Vizing's Theorem says that the edge chromatic number of a simple graph $G$ is equal to $\Delta$ or $\Delta+1$. $G$ is class one if $\chi^{\prime}(G)=\Delta$ and class two if $\chi^{\prime}(G)=\Delta+1$. A class two graph is critical if $\chi^{\prime}(G-e)<\chi^{\prime}(G)$ for any edge $e$ of $G$. A critical graph $G$ is $\Delta$-critical if it has maximum degree $\Delta$.

\footnotetext{
${ }^{*}$ Research supported by the Foundation of Zhejiang Educational Committee (No.Y201226078).

${ }^{\dagger}$ Corresponding author. Research supported by NSFC (No. 11071223), ZJNSFC (No. Z6090150), ZJIP (No. T200905), ZSDZZZZXK13, and IP-OCNS-ZJNU.
} 
In 1965, Vizing[6] proposed the following well-known Planar Graph Coloring conjecture:

Conjecture 1 Every planar graph $G$ with $\Delta=6,7$ is class one.

The case $\Delta=7$ was confirmed independently by Sanders and Zhao [4], and by Zhang [9]. This result was further extended by Sanders and Zhao [5] to a graph with $\Delta=7$ which can be embedded in a surface of characteristic zero. The case $\Delta=6$ remains open.

$\operatorname{In}[10]$, Zhou proved that every planar graph $G$ with $\Delta=6$ is class one if it is $C_{3}$-free, or $C_{4}$-free, or $C_{5}$-free. Li, Luo and $\mathrm{Niu}[2]$ generalized Zhou's results to the surface of Euler characteristic at least -3 or -1 . Bu and Wang[1] proved that planar graphs $G$ with $\Delta=6$ and without 6 -cycles, or without two adjacent 3 -cycles are class one. Wang and Chen [7] proved that planar graphs with $\Delta=6$ and without a 5 -cycle with a chord is class one. More recently, Wang, Chen and Wang [8] further proved that planar graphs with $\Delta=6$ and without a 6-cycle with a chord is class one.

In this paper, we prove the following result, which extends a result in [1] and [10]:

Theorem 1. If $G$ is a planar graph with $\Delta=6$ and without 7 -cycles, then $\chi^{\prime}(G)=\Delta$.

To show Theorem 1, we need to introduce some notation. For $f \in F(G)$, we use $b(f)$ to denote the boundary walk of $f$ and write $f=\left[u_{1} u_{2} \cdots u_{k}\right]$ if $u_{1}, u_{2}, \ldots, u_{k}$ are the vertices of $b(f)$ in a cyclic order. For $x \in V(G) \cup F(G)$, let $d(x)$ denote the degree of $x$ in $G$. A vertex of degree $k$ (at least $k$, at most $k$, respectively) is called a $k$-vertex (or $k^{+}$-vertex, $k^{-}$-vertex, respectively). Similarly, we can define a $k$-face, $k^{+}$-face and $k^{-}$-face. Let $v \in V(G)$. If a $k$-vertex $u$ is adjacent to $v$, then $u$ is called a $k$-neighbor of $v$, and we use $d_{k}(v)$ to denote the number of $k$-neighbors of $v$. Similarly, we can define $d_{k^{+}}(v)$ and $d_{k^{-}}(v)$. For $i \geqslant 3$, let $m_{i}(v)$ denote the number of $i$-faces incident to $v$. Moreover, $m_{i^{+}}(v)$ and $m_{i^{-}}(v)$ can be defined analogously. Let $N(v)$ denote the set of neighbors of $v$, and let $N[v]=N(v) \cup\{v\}$. For $S \subseteq V(G)$, let $N(S)=\cup_{u \in S} N(u)$.

The following is the outstanding Vizing's Adjacent Lemma (we denote it by VAL for short).

Lemma 2. ([6]) If $G$ is a $\Delta$-critical graph and $x y$ is an edge of $G$, then $d(x)+d(y) \geqslant \Delta+2$ and $x$ is adjacent to at least $(\Delta-d(y)+1) \Delta$-vertices. Furthermore, every vertex is adjacent to at least two $\Delta$-vertices.

Let $G$ be a 6 -critical graph and $v \in V(G)$. Then the assertions (P1) to (P5) below follow automatically from Lemma 2 .

(P1) If $d(v)=2$, then $d_{6}(v)=2$.

(P2) If $d(v)=3$, then $d_{4^{-}}(v)=0$ and $d_{5}(v) \leqslant 1$.

(P3) If $d(v)=4$, then $d_{3^{-}}(v)=0$ and $d_{4}(v) \leqslant 1$; and if $d_{4}(v)=1$, then $d_{6}(v)=3$.

(P4) If $d(v)=5$, then $d_{2}(v)=0$ and $d_{3}(v) \leqslant 1$; moreover, $d_{6}(v)=4$ if $d_{3}(v)=1$, and $d_{6}(v) \geqslant 3$ if $d_{4}(v) \geqslant 1$.

(P5) If $d(v)=6$, then $d_{2}(v) \leqslant 1$; moreover, $d_{6}(v)=5$ if $d_{2}(v)=1, d_{6}(v) \geqslant 4$ if $d_{3}(v) \geqslant 1$, and $d_{6}(v) \geqslant 3$ if $d_{4}(v) \geqslant 1$. 


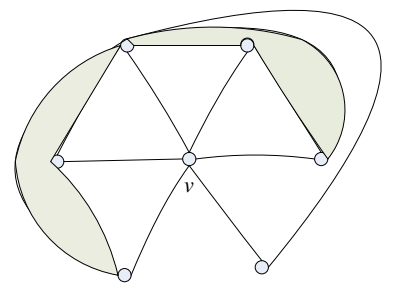

$G_{0}$

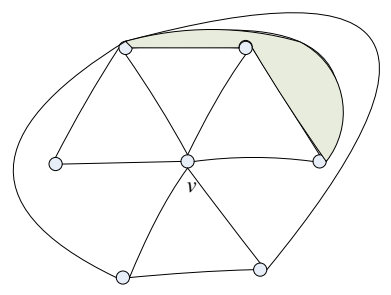

$G_{1}$

Figure 1: The configurations in Claim 5, where $v$ is a bad 6-vertex.

The vertex $v$ is called bad if either $d(v)=6, m_{3}(v)=4$ and $m_{4}(v)=2$, or $d(v)=$ $m_{3}(v)=5$ and $d_{6}(v)=2$, or $d(v)=m_{3}(v)=4$. It is easy to see that a bad 5-vertex $v$ satisfies $d_{5}(v)=3$ by $(\mathrm{P} 4)$.

Lemma 3. ( [9]) Let $G$ be a $\Delta$-critical graph. If $x y \in E(G)$ and $d(x)+d(y)=\Delta+2$, then the following hold:

(1) Every vertex in $N(x, y) \backslash\{x, y\}$ is a $\Delta$-vertex.

(2) Every vertex in $N(N(x, y)) \backslash\{x, y\}$ is of degree at least $\Delta-1$.

(3) If $d(x), d(y)<\Delta$, then every vertex in $N(N(x, y)) \backslash\{x, y\}$ is a $\Delta$-vertex.

Lemma 4. ([3]) Let $G$ be a critical graph and $x$ be a 3-vertex in $G$. If $x$ is adjacent to three $\Delta$-vertices, then at least one $\Delta$-vertex in $N(x)$ is adjacent to only one $(\Delta-1)^{-}$-vertex which is $x$.

\section{Proof of Theorem 1.1}

Let $G$ be a planar graph with $\Delta=6$ and without 7 -cycles that is embedded in the plane. Assume to the contrary that $G$ is class two. Without loss of generality, we may assume that $G$ is 6 -critical. Then $G$ is 2 -connected, implying that the boundary of each face forms a cycle and every edge lies on the boundaries of two faces.

We first investigate structural properties of $G$ and then use Euler's formula and the discharging technique to derive a contradiction. Given a $k$-vertex $v \in V(G)$, let $v_{0}, v_{1}, \cdots$, $v_{k-1}$ be the neighbors of $v$ in clockwise order. For $0 \leqslant i \leqslant k-1$, let $f_{i}$ be the incident face of $v$ with $v v_{i}$ and $v v_{i+1}$ as boundary edges, where indices are taken modulo $k$.

Claim 5. Let $v$ be a 6-vertex with $m_{3}(v)=4$. Then one of the following cases holds:

(1) $v$ is bad such that $G(N[v]) \cong G_{0}$ or $G_{1}$, as shown in Figure 1;

(2) $m_{4}(v)=1$ and $m_{8^{+}}(v)=1$;

(3) $m_{5}(v)=1$ and $m_{6^{+}}(v)=1$;

(4) $m_{6^{+}}(v)=2$.

Proof. Since $m_{3}(v)=4$, the proof is split into the following three cases by symmetry. 
Case $1 d\left(f_{i}\right)=3$ for $i=1,2,3,4$.

Since $G$ contains no 7 -cycles, it is easy to see that $v_{0} v_{1}, v_{0} v_{5} \notin E(G)$. Let $f_{5}=$ $\left[v v_{5} y_{1} \cdots y_{p} v_{0}\right]$ and $f_{0}=\left[v v_{0} z_{1} \cdots z_{q} v_{1}\right]$. Then $p, q \geqslant 1$ and $p, q \neq 4$. By symmetry, we may assume that $q \geqslant p$. If $p \geqslant 3$, then (4) holds. If $p=2$ and $q \geqslant 3$, then (3) holds. If $p=1$ and $q \geqslant 5$, then (2) holds. Otherwise, it suffices to consider the following two subcases to derive (1):

Subcase $1.1 p=1$ and $1 \leqslant q \leqslant 3$.

Since $G$ is $C_{7}$-free, it follows that $y_{1} \in\left\{v_{2}, v_{3}, v_{4}\right\}$.

(1.1a) $y_{1}=v_{4}$, implying that $d\left(v_{5}\right)=2$.

- Assume that $q=1$. Then $z_{1} \in\left\{v_{2}, v_{3}, v_{4}\right\}$ as before. If $z_{1}=v_{2}$, then $d\left(v_{1}\right)=2$. If $z_{1}=v_{4}$, then $d\left(v_{0}\right)=2$. Thus, $N(v)$ has at least two 2-vertices in both cases, contradicting (P5). If $z_{1}=v_{3}$, we get a 7 -cycle $C_{7}=v v_{5} v_{4} v_{0} v_{3} v_{2} v_{1} v$, a contradiction.

- Assume that $q=2$. Then at least one of $z_{1}, z_{2}$ belongs to $\left\{v_{2}, v_{3}\right\}$ since otherwise we get a 7 -cycle $C_{7}=v v_{0} z_{1} z_{2} v_{1} v_{2} v_{3} v$. If $z_{2}=v_{2}$, then $d\left(v_{1}\right)=2$, contradicting (P5). If $z_{2}=$ $v_{3}$, then $z_{1} \neq v_{4}$, and we get $C_{7}=v_{0} z_{1} v_{3} v_{2} v_{1} v v_{4} v_{0}$. If $z_{1}=v_{2}$, we get $C_{7}=v v_{1} z_{2} v_{2} v_{3} v_{4} v_{5} v$. If $z_{1}=v_{3}$, we have two possibilities. When $z_{2}=v_{2}$, we have $d\left(v_{1}\right)=2$, contradicting (P5). When $z_{2} \neq v_{2}$, we get $C_{7}=v_{1} z_{2} v_{3} v_{4} v_{5} v v_{2} v_{1}$.

- Assume that $q=3$. Then $v_{2} \in\left\{z_{1}, z_{2}, z_{3}\right\}$, for otherwise we take $C_{7}=v v_{0} z_{1} z_{2} z_{3} v_{1} v_{2} v$. If $v_{2}=z_{3}$, then $d\left(v_{1}\right)=2$, contradicting (P5). If $v_{2}=z_{2}$, we take $C_{7}=v v_{1} z_{3} v_{2} v_{3} v_{4} v_{5} v$. If $v_{2}=z_{1}$, then $z_{2}, z_{3} \notin N(v)$ by the planarity of $G$, hence we have $C_{7}=v v_{1} z_{3} z_{2} v_{2} v_{3} v_{4} v$.

(1.1b) $y_{1}=v_{3}$.

- Assume that $q=1$. Then $z_{1} \in\left\{v_{2}, v_{3}\right\}$ by the planarity of $G$ and the hypothesis that $G$ is $C_{7}$-free. If $z_{1}=v_{2}$, we get $C_{7}=v v_{1} v_{2} v_{0} v_{3} v_{4} v_{5} v$. If $z_{1}=v_{3}$, we have $G(N[v]) \cong G_{0}$, hence the conclusion (1) holds.

- Assume that $q=2$. We note that at least one of $z_{1}, z_{2}$ belongs to $\left\{v_{2}, v_{3}\right\}$ by the planarity of $G$ and the fact that $G$ is $C_{7}$-free. If $z_{1}=v_{2}$, we get $C_{7}=v v_{1} z_{2} v_{2} v_{3} v_{4} v_{5} v$. If $z_{1}=v_{3}$, we assert that $z_{2} \neq v_{2}$, otherwise it follows that $d\left(v_{1}\right)=2$ and $d\left(v_{3}\right)=3$, contradicting (P5). Thus, we get $C_{7}=v v_{2} v_{1} z_{2} v_{3} v_{4} v_{5} v$. If $z_{2}=v_{2}$, we derive by (P5) that $z_{1} \neq v_{3}$, hence $C_{7}=v v_{0} z_{1} v_{2} v_{3} v_{4} v_{5} v$. If $z_{2}=v_{3}$, it is easy to deduce that $d\left(v_{3}\right) \geqslant 7$, contradicting the assumption that $\Delta=6$.

- Assume that $q=3$. Then $v_{2} \in\left\{z_{1}, z_{2}, z_{3}\right\}$ since $G$ is $C_{7}$-free. If $z_{3}=v_{2}$, then $z_{2} \neq v_{3}$ by (P5). When $z_{1} \neq v_{3}$, we get $C_{7}=v v_{0} z_{1} z_{2} v_{2} v_{3} v_{4} v$. When $z_{1}=v_{3}$, we get $C_{7}=v v_{1} v_{2} z_{2} v_{3} v_{4} v_{5} v$. If $z_{2}=v_{2}$, we get $C_{7}=v v_{1} z_{3} v_{2} v_{3} v_{4} v_{5} v$. If $z_{1}=v_{2}$, we get $C_{7}=v v_{1} z_{3} z_{2} v_{2} v_{3} v_{4} v$.

(1.1c) $y_{1}=v_{2}$.

- If $q=1$, then $z_{1}=v_{2}$ by the planarity of $G$ and the hypothesis that $G$ is $C_{7}$-free. Hence $d\left(v_{1}\right)=d\left(v_{0}\right)=2$, contradicting (P5).

- Assume that $q=2$. By the planarity of $G, v_{3} \notin\left\{z_{1}, z_{2}\right\}$. If $v_{2} \notin\left\{z_{1}, z_{2}\right\}, G$ contains a 7-cycle $C_{7}=v v_{0} z_{1} z_{2} v_{1} v_{2} v_{3} v$. If $z_{1}=v_{2}$, we get $C_{7}=v v_{1} z_{2} v_{2} v_{3} v_{4} v_{5} v$. If $z_{2}=v_{2}$, we get $C_{7}=v v_{0} z_{1} v_{2} v_{3} v_{4} v_{5} v$. 
- Assume that $q=3$. Then $v_{2} \in\left\{z_{1}, z_{2}, z_{3}\right\}$ since $G$ is $C_{7}$-free. If $z_{3}=v_{2}$, we get $C_{7}=v v_{0} z_{1} z_{2} v_{2} v_{3} v_{4} v$. If $z_{2}=v_{2}$, we get $C_{7}=v v_{1} z_{3} v_{2} v_{3} v_{4} v_{5} v$. If $z_{1}=v_{2}$, we get $C_{7}=v v_{1} z_{3} z_{2} v_{2} v_{3} v_{4} v$.

Subcase $1.2 p=q=2$.

Since $G$ is $C_{7}$-free, we see that at least one of $y_{1}, y_{2}$ belongs to $\left\{v_{3}, v_{4}\right\}$, and at least one of $z_{1}, z_{2}$ belongs to $\left\{v_{2}, v_{3}\right\}$. Furthermore, $y_{2} \neq v_{1}$ and $z_{1} \neq v_{5}$.

- If $y_{2}=v_{4}$, then we get $C_{7}=v v_{5} y_{1} v_{4} v_{3} v_{2} v_{1} v$. If $z_{1}=v_{2}$, we have a similar proof. So assume that $z_{1} \neq v_{2}$.

- Assume that $y_{1}=v_{4}$. Then $d\left(v_{5}\right)=2$. If $y_{2}=v_{3}$, then $d\left(v_{4}\right)=3$, contradicting (P5). If $y_{2}=v_{2}$, then $z_{2}=v_{2}$ and $d\left(v_{1}\right)=2$, contradicting (P5). If $y_{2} \notin\left\{v_{2}, v_{3}\right\}$, we get $C_{7}=v v_{0} y_{2} v_{4} v_{3} v_{2} v_{1} v$.

If $z_{2}=v_{2}$, we have a similar proof. Hence assume that $z_{2} \neq v_{2}$.

- Assume that $y_{1}=v_{3}$. If $z_{2}=v_{3}$, then $d\left(v_{3}\right) \geqslant 7$, a contradiction. If $z_{1}=v_{3}$, then we get $C_{7}=v v_{2} v_{1} z_{2} v_{3} v_{4} v_{5} v$.

- Assume that $y_{2}=v_{3}$. Then we get $C_{7}=v v_{1} v_{2} v_{3} y_{1} v_{5} v_{4} v$.

Case $2 d\left(f_{i}\right)=3$ for $i=1,2,3,5$.

Since $G$ is $C_{7}$-free, we see $v_{0} v_{1}, v_{0} v_{4}, v_{1} v_{5}, v_{4} v_{5} \notin E(G)$. Let $f_{4}=\left[v v_{4} y_{1} \cdots y_{p} v_{5}\right]$ and $f_{0}=\left[v v_{0} z_{1} \cdots z_{q} v_{1}\right]$ such that $q \geqslant p \geqslant 1$ and $p, q \neq 4$. If one of (2)-(4) holds, we are done. Otherwise, similar to Case 1, it suffices to consider the following two subcases:

Subcase $2.1 p=1$ and $1 \leqslant q \leqslant 3$.

Since $G$ is $C_{7}$-free, it follows that $y_{1} \in\left\{v_{2}, v_{3}\right\}$.

(2.1a) $y_{1}=v_{3}$, implying that $d\left(v_{4}\right)=2$. Then $z_{1} \neq v_{5}$, for otherwise $d\left(v_{0}\right)=2$, contradicting (P5).

- Assume that $q=1$. Then $z_{1} \in\left\{v_{2}, v_{3}\right\}$ by the same reason. If $z_{1}=v_{2}$, then $d\left(v_{1}\right)=2$, contradicting (P5). If $z_{1}=v_{3}$, then $G(N[v]) \cong G_{1}$, hence (1) holds.

- Assume that $q=2$. Since $G$ is $C_{7}$-free, at least one of $z_{1}, z_{2}$ coincides with $v_{2}$ or $v_{3}$. If $z_{2}=v_{2}$, then $d\left(v_{1}\right)=2$, contradicting (P5). If $z_{1}=v_{2}$, we get $C_{7}=v v_{0} v_{5} v_{3} v_{2} z_{2} v_{1} v$. If $z_{2}=v_{3}$, we get $C_{7}=v v_{5} v_{0} z_{1} v_{3} v_{2} v_{1} v$. If $z_{1}=v_{3}$, we get $C_{7}=v v_{0} v_{5} v_{3} z_{2} v_{1} v_{2} v$.

- Assume that $q=3$. Then $v_{2} \in\left\{z_{1}, z_{2}, z_{3}\right\}$ by the previous analysis. If $z_{3}=v_{2}$, then $d\left(v_{1}\right)=2$, contradicting (P5). If $z_{2}=v_{2}$, we get $C_{7}=v v_{0} v_{5} v_{3} v_{2} z_{3} v_{1} v$. If $z_{1}=v_{2}$, we get $C_{7}=v v_{5} v_{3} v_{2} z_{2} z_{3} v_{1} v$.

(2.1b) $y_{1}=v_{2}$.

- Assume that $q=1$. By the planarity of $G$ and the foregoing argument, $z_{1} \notin$ $N(v) \backslash\left\{v_{2}\right\}$. If $z_{1}=v_{2}$, we have $G(N[v]) \cong G_{1}$ and therefore (1) holds.

- Assume that $q=2$. It follows that $v_{2} \in\left\{z_{1}, z_{2}\right\}$ since $G$ is $C_{7}$-free. If $z_{1}=v_{2}$, then $d\left(v_{2}\right) \geqslant 7$, a contradiction. If $z_{2}=v_{2}$, then $z_{1} \neq v_{5}$, and we get $C_{7}=v v_{5} v_{0} z_{1} v_{2} v_{3} v_{4} v$.

- Assume that $q=3$. Then $v_{2} \in\left\{z_{1}, z_{2}, z_{3}\right\}$. If $z_{1}=v_{2}$, we get $C_{7}=v v_{1} z_{3} z_{2} v_{2} v_{3} v_{4} v$. If $z_{3}=v_{2}$, we get $C_{7}=v v_{0} z_{1} z_{2} v_{2} v_{3} v_{4} v$. If $z_{2}=v_{2}$, we have two possibilities. When $z_{1} \neq v_{5}$, we get $C_{7}=v v_{5} v_{0} z_{1} v_{2} v_{3} v_{4} v$. When $z_{1}=v_{5}$, we have $d\left(v_{0}\right)=2$ and $d\left(v_{5}\right)=3$, contradicting (P5). 
Subcase $2.2 p=q=2$.

We see that at least one of $y_{1}, y_{2}\left(z_{1}, z_{2}\right.$, respectively) coincides with $v_{2}$ or $v_{3}$.

- Assume that $y_{1}=v_{3}$. Then $d\left(v_{4}\right)=2$. By the previous discussion, $y_{2} \neq v_{1}$. If $y_{2}=v_{0}$, then $d\left(v_{5}\right)=2$, contradicting (P5). If $y_{2}=v_{2}$, then $d\left(v_{3}\right)=3$, contradicting (P5). This shows that $y_{2} \notin\left\{v_{0}, v_{1}, v_{2}\right\}$, hence we get $C_{7}=v v_{0} v_{5} y_{2} v_{3} v_{2} v_{1} v$.

If $z_{2}=v_{2}$, we have a similar proof.

- Assume that $y_{2}=v_{3}$. If $z_{1}=v_{2}$, we get $C_{7}=v v_{1} z_{2} v_{2} v_{3} y_{1} v_{4} v$. If $z_{1}=v_{3}$, we get $C_{7}=v v_{4} y_{1} v_{3} z_{2} v_{1} v_{2} v$. If $z_{2}=v_{3}$, we claim that $z_{1} \neq v_{5}$, for otherwise $d\left(v_{0}\right)=2$ and $d\left(v_{5}\right)=3$, contradicting (P5). Thus, we get $C_{7}=v v_{5} v_{0} z_{1} v_{3} y_{1} v_{4} v$.

If $z_{1}=v_{2}$, we have a similar proof.

- Assume that one of $y_{1}, y_{2}$ coincides with $v_{2}$. By the planarity of $G$, none of $z_{1}, z_{2}$ coincides with $v_{3}$. Thus, we get $C_{7}=v v_{0} z_{1} z_{2} v_{1} v_{2} v_{3} v$.

Case $3 d\left(f_{i}\right)=3$ for $i=1,2,4,5$.

Since $G$ is $C_{7}$-free, we see that $v_{0} v_{1}, v_{0} v_{3}, v_{1} v_{4}, v_{3} v_{4} \notin E(G)$. Let $f_{3}=\left[v v_{3} y_{1} \cdots y_{p} v_{4}\right]$ and $f_{0}=\left[v v_{0} z_{1} \cdots z_{q} v_{1}\right]$ such that $q \geqslant p \geqslant 1$ and $p, q \neq 4$. If one of (2)-(4) holds, we are done. Otherwise, it suffices to consider the following two subcases:

Subcase $3.1 p=1$ and $1 \leqslant q \leqslant 3$.

Since $G$ is $C_{7}$-free, it follows that $y_{1} \in\left\{v_{2}, v_{5}\right\}$. Without loss of generality, we assume that $y_{1}=v_{2}$. Then $d\left(v_{3}\right)=2$.

- If $q=1$, then $z_{1} \in\left\{v_{2}, v_{5}\right\}$ similarly, so $d\left(v_{1}\right)=2$ or $d\left(v_{0}\right)=2$, contradicting (P5).

- Assume that $q=2$. We note that $v_{3} \notin\left\{z_{1}, z_{2}\right\}$ by the plane embedding of $G$. Thus, $v_{2} \in\left\{z_{1}, z_{2}\right\}$. If $z_{1}=v_{2}$, we get $C_{7}=v v_{0} v_{5} v_{4} v_{2} z_{2} v_{1} v$. If $z_{2}=v_{2}$, then $d\left(v_{1}\right)=2$, contradicting (P5).

- Assume that $q=3$. Then $v_{2} \in\left\{z_{1}, z_{2}, z_{3}\right\}$. If $z_{3}=v_{2}$, then $d\left(v_{1}\right)=2$, contradicting (P5). If $z_{1}=v_{2}$, we get $C_{7}=v v_{5} v_{4} v_{2} z_{2} z_{3} v_{1} v$. If $z_{2}=v_{2}$, we get $C_{7}=v v_{0} v_{5} v_{4} v_{2} z_{3} v_{1} v$.

Subcase $3.2 p=q=2$.

If $y_{1}, y_{2} \notin\left\{v_{2}, v_{5}\right\}$, we get $C_{7}=v v_{2} v_{3} y_{1} y_{2} v_{4} v_{5} v$. If $z_{1}, z_{2} \notin\left\{v_{2}, v_{5}\right\}$, we get a similar 7-cycle. Otherwise, by symmetry, we assume that the following two possibilities:

- Assume that $y_{1}=v_{2}$. Then $d\left(v_{3}\right)=2$. Obviously, $y_{2} \neq v_{1}$ since $v_{1} v_{4} \notin E(G)$. If $y_{2}=v_{5}$, then $d\left(v_{4}\right)=2$, contradicting (P5). If $y_{2} \neq v_{0}$, we get $C_{7}=v v_{0} v_{5} v_{4} y_{2} v_{2} v_{1} v$. So suppose that $y_{2}=v_{0}$. In this case, we must have $v_{2} \in\left\{z_{1}, z_{2}\right\}$. If $z_{2}=v_{2}$, then $d\left(v_{1}\right)=2$, contradicting (P5). If $z_{1}=v_{2}$, we get $C_{7}=v v_{1} z_{2} v_{2} v_{0} v_{5} v_{4} v$.

- Assume that $y_{2}=v_{2}$. We get $C_{7}=v v_{3} y_{1} v_{2} v_{4} v_{5} v_{0} v$.

For a bad 6-vertex $v$, Claim 5 asserts that $G(N[v]) \cong G_{0}$ or $G_{1}$, see Figure 1 . We say that a 6-vertex in the induced subgraph $G[N(v)]$ other than $v$ is a master of $v$, and $v$ is the slave of its master. Clearly, if $u$ is a master of some 6 -vertex, then $m_{3}(u)=2$, $m_{4}(u)=2$, and $u$ has only one slave.

Claim 6. Let $v$ be a 6-vertex with $m_{3}(v)=3$. If $m_{4}(v)=3$, then $d_{3^{-}}(v)=0$. 
Proof. Assume to the contrary that $d_{3^{-}}(v)>0$. If $f_{i}$ is a 4 -face, we let $f_{i}=\left[v v_{i} y_{i} v_{i+1}\right]$, where indices are taken modulo 6 .

Case $1 d\left(f_{i}\right)=3$ for $i=1,3,5$.

Then $d\left(f_{j}\right)=4$ for $j=0,2,4$. We note that if $y_{j} \in\left\{v_{j-1}, v_{j+2}\right\}$, then $d\left(v_{j}\right)=2$ or $d\left(v_{j+1}\right)=2$ for each $j=0,2,4$. By (P5), at least two of $y_{j}$ 's, say $y_{2}$ and $y_{4}$, do not coincide with $v_{j-1}$ and $v_{j+2}$. If $y_{2}=v_{5}$, we get $C_{7}=v v_{1} v_{2} v_{5} y_{4} v_{4} v_{3} v$. If $y_{4}=v_{2}$, we have a similar proof. If $y_{2} \neq y_{4}$, we get $C_{7}=v v_{2} y_{2} v_{3} v_{4} y_{4} v_{5} v$. Assume that $y_{2}=y_{4}$, which implies that $y_{2} \notin\left\{v_{0}, v_{1}\right\}$. If $y_{0} \in\left\{v_{2}, v_{5}\right\}$, say $y_{0}=v_{2}$, then we get $C_{7}=v v_{5} v_{0} v_{2} y_{2} v_{3} v_{4} v$. Otherwise, $y_{0} \notin\left\{v_{2}, v_{5}\right\}$, we have two possibilities. If $y_{0} \neq y_{2}$, then we get $C_{7}=v v_{0} y_{0} v_{1} v_{2} y_{2} v_{3} v$. If $y_{0}=y_{2}$, i.e., $y_{0}, y_{2}, y_{4}$ identify to one vertex, then it is easy to see that $d\left(v_{i}\right) \geqslant 3$ for all $i=0,1, \cdots, 5$. Since $d_{3^{-}}(v)>0$, we may assume, without loss of generality, that $d\left(v_{1}\right)=3$. By $(\mathrm{P} 2), d\left(v_{2}\right) \geqslant 5$. However, $v_{2}$ is a cut vertex of $G$, contradicting the fact that $G$ is 2-connected.

Case $2 d\left(f_{i}\right)=3$ for $i=1,2,4$.

Then $d\left(f_{j}\right)=4$ for $j=0,3,5$. If $y_{3} \notin N(v)$, we get $C_{7}=v v_{1} v_{2} v_{3} y_{3} v_{4} v_{5} v$. So assume that $y_{3} \in N(v)$. We have the following subcases:

- If $y_{3}=v_{0}$, we get $C_{7}=v v_{1} v_{2} v_{3} v_{0} v_{4} v_{5} v$.

- Assume that $y_{3}=v_{5}$, then $d\left(v_{4}\right)=2$. If $y_{0} \notin N(v)$, we get $C_{7}=v v_{0} y_{0} v_{1} v_{2} v_{3} v_{5} v$. Otherwise, $y_{0} \in\left\{v_{2}, v_{3}, v_{5}\right\}$ by the plane embedding of $G$. If $y_{0}=v_{2}$, then $d\left(v_{1}\right)=2$, contradicting (P5). If $y_{0}=v_{5}$, then it follows that $y_{5} \notin N(v)$ by the plane embedding of $G$, hence we construct $C_{7}=v v_{0} y_{5} v_{5} v_{3} v_{2} v_{1} v$. So assume that $y_{0}=v_{3}$. If $y_{5} \neq v_{3}$, we get $C_{7}=v v_{0} y_{5} v_{5} v_{3} v_{2} v_{1} v$. If $y_{5}=v_{3}$, then $d\left(v_{0}\right)=2$, contradicting (P5).

- Assume that $y_{3}=v_{2}$. Then $d\left(v_{3}\right)=2$. If $y_{5} \notin N(v)$, we get $C_{7}=v v_{0} y_{5} v_{5} v_{4} v_{2} v_{1} v$. Otherwise, we have $y_{5} \in\left\{v_{1}, v_{2}, v_{4}\right\}$ by the planarity of $G$. If $y_{5}=v_{4}$, then $d\left(v_{5}\right)=2$, contradicting (P5). If $y_{5}=v_{1}$, or $y_{5}=v_{2}$, then $y_{0} \notin N(v)$ by (P5) and the planarity of $G$, thus we have $C_{7}=v v_{0} y_{0} v_{1} v_{2} v_{4} v_{5} v$.

- Assume that $y_{3}=v_{1}$. If $y_{5} \notin N(v)$, we get $C_{7}=v v_{0} y_{5} v_{5} v_{4} v_{1} v_{2} v$. Otherwise, $y_{5} \in\left\{v_{1}, v_{4}\right\}$ by the planarity of $G$. If $y_{5}=v_{4}$, then $d\left(v_{5}\right)=2$. When $y_{0}=v_{4}$, we have $d\left(v_{0}\right)=2$, contradicting (P5). When $y_{0} \neq v_{4}$, we get $C_{7}=v v_{4} v_{0} y_{0} v_{1} v_{2} v_{3} v$. If $y_{5}=v_{1}$, then it is easy to see that $y_{0} \notin N(v)$, and hence $d\left(v_{1}\right) \geqslant 7$, a contradiction.

Case $3 d\left(f_{i}\right)=3$ for $i=1,2,3$.

Then $d\left(f_{j}\right)=4$ for $j=0,4,5$. If $y_{4} \notin N(v)$, we get $C_{7}=v v_{5} y_{4} v_{4} v_{3} v_{2} v_{1} v$. Otherwise, it suffices to handle the case $y_{4} \in\left\{v_{0}, v_{1}, v_{2}, v_{3}\right\}$.

- If $y_{4}=v_{0}$, we get $C_{7}=v v_{1} v_{2} v_{3} v_{4} v_{0} v_{5} v$. If $y_{0}=v_{5}$, we have a similar proof.

- Assume that $y_{4}=v_{3}$, then $d\left(v_{4}\right)=2$. If $y_{5} \notin N(v)$, we get $C_{7}=v v_{0} y_{5} v_{5} v_{3} v_{2} v_{1} v$. Otherwise, suppose that $y_{5} \in\left\{v_{1}, v_{2}, v_{3}\right\}$ by the plane embedding of $G$. If $y_{5}=v_{3}$, then $d\left(v_{5}\right)=2$, contradicting (P5). If $y_{5}=v_{1}$, then $y_{0} \notin N(v)$, we get $C_{7}=v v_{0} y_{0} v_{1} v_{2} v_{3} v_{4} v$. Finally, suppose that $y_{5}=v_{2}$. If $y_{0}=v_{2}$, then $d\left(v_{0}\right)=2$, contradicting (P5). Otherwise, we can construct a 7 -cycle as above.

If $y_{0}=v_{2}$, we have a similar proof. 
- Assume that $y_{4}=v_{1}$. If $y_{5} \notin N(v)$, we get $C_{7}=v v_{0} y_{5} v_{5} v_{1} v_{2} v_{3} v$. Otherwise, $y_{5}=v_{1}$ by the planarity of $G$. Noting that $y_{0} \notin N(v)$, we can construct a 7-cycle as above.

If $y_{0}=v_{4}$, we have a similar proof.

- If $y_{4}=v_{2}$, then $y_{0}=v_{3}$ by symmetry, which is impossible by the planarity of $G$.

Claim 7. If $v$ is a 6-vertex adjacent to a bad 5-vertex, then $m_{8^{+}}(v) \geqslant 2$.

Proof. Assume that $v_{0}, v_{1}, \ldots, v_{5}$ are the neighbors of $v$ in clockwise order, and $v_{1}$ is a bad 5 -vertex. Then $m_{3}\left(v_{1}\right)=5, d_{6}\left(v_{1}\right)=2$ and $d_{5}\left(v_{1}\right)=3$ by definition. This implies that $v_{1} v_{2}, v_{1} v_{0} \in E(G)$. Let $x$ and $y$ be the other two neighbors of $v_{1}$ such that $v, v_{0}, x, y, v_{2}$ are arranged around $v$ in clockwise order. The proof is split into three cases as follows:

Case $1 v_{3}=x$.

Since $G$ is $C_{7}$-free, it is easy to inspect that neither $v_{4}$ nor $v_{5}$ is adjacent to a vertex in $\left\{x, v_{0}\right\}$. Moreover, since $G$ is simple and $G$ is embedded in the plane, both $v_{4}$ and $v_{5}$ can not identify to $x$, hence $v_{1} v_{4}, v_{1} v_{5} \notin E(G)$. Our goal is to show that $d\left(f_{i}\right) \geqslant 8$ for $i=3,5$.

Let $f_{3}=\left[v x u_{1} \cdots u_{s} v_{4}\right]$. Obviously, $d\left(f_{3}\right)=s+3 \geqslant 4$, and $v_{1}, v_{2}, y \notin\left\{u_{1}, u_{2}, \cdots, u_{s}\right\}$. If $s=1$, we get $C_{7}=v v_{4} u_{1} x v_{1} y v_{2} v$. If $s=2$, we get $C_{7}=v v_{4} u_{2} u_{1} x y v_{2} v$. If $s=3$, we get $C_{7}=v v_{4} u_{3} u_{2} u_{1} x v_{1} v$. Since $G$ is $C_{7}$-free, $s \neq 4$. Therefore, $s \geqslant 5$, that is $d\left(f_{3}\right) \geqslant 8$.

Let $f_{5}=\left[v v_{5} z_{1} \cdots z_{l} v_{0}\right]$. Then $d\left(f_{5}\right)=l+3 \geqslant 4, l \neq 4$, and $v_{1}, v_{2}, y \notin\left\{z_{1}, z_{2}, \cdots, z_{l}\right\}$. If $l=1$, we get $C_{7}=v v_{5} z_{1} v_{0} v_{1} y v_{2} v$. If $l=2$, we get $C_{7}=v v_{5} z_{1} z_{2} v_{0} v_{1} v_{2} v$. If $l=3$, we get $C_{7}=v v_{5} z_{1} z_{2} z_{3} v_{0} v_{1} v$. Therefore, $s \geqslant 5$, that is $d\left(f_{3}\right) \geqslant 8$.

If $v_{5}=y$, we have a similar discussion.

Case $2 v_{3}=y$.

By Case 1 , we assume that $v_{5} \neq y$. We are going to show that $d\left(f_{i}\right) \geqslant 8$ for $i=2,5$.

Firstly, we show that $d\left(f_{2}\right) \geqslant 8$. If $d\left(f_{2}\right)=3$, then $d\left(v_{2}\right)=3$, contradicting the definition of a bad 5-vertex. So assume that $f_{2}=\left[v v_{2} u_{1} \cdots u_{s} y\right]$, where $s \geqslant 1$ and $s \neq 4$. Note that $v_{0}, v_{1}, x \notin\left\{u_{1}, u_{2}, \cdots, u_{s}\right\}$. If $s=1$, we get $C_{7}=v v_{0} x y u_{1} v_{2} v_{1} v$. If $s=2$, we get $C_{7}=v v_{1} x y u_{2} u_{1} v_{2} v$. If $s=3$, we get $C_{7}=v v_{1} y u_{3} u_{2} u_{1} v_{2} v$. This shows that $s \geqslant 5$, namely $d\left(f_{2}\right) \geqslant 8$.

Secondly, we show that $d\left(f_{5}\right) \geqslant 8$. If $v_{5}=x$, then we can show that $d\left(f_{5}\right) \geqslant 8$ as above. Otherwise, assume that $v_{5} \neq x$. Since $v_{0} v_{5} \notin E(G)$, we see that $d\left(f_{5}\right) \geqslant 4$. Again, let $f_{5}=\left[v v_{5} z_{1} \cdots z_{l} v_{0}\right]$ with $l \geqslant 1$. Then $v_{1}, v_{2} \notin\left\{z_{1}, z_{2}, \cdots, z_{l}\right\}$ by the planarity of $G$. It suffices to inspect that $l \notin\{1,2,3\}$. In fact, if $l=3$, we get $C_{7}=v v_{5} z_{1} z_{2} z_{3} v_{0} v_{1} v$. If $l=2$, we get $C_{7}=v v_{5} z_{1} z_{2} v_{0} v_{1} v_{2} v$. So assume that $l=1$. Since $x v_{5} \notin E(G)$, we derive that $z_{1} \neq x$. We get $C_{7}=v v_{5} z_{1} v_{0} x v_{1} v_{2} v$.

If $v_{5}=x$, we have a similar proof.

Case $3 v_{3}, v_{5} \notin\{x, y\}$.

To show that $d\left(f_{i}\right) \geqslant 8$ for $i=2,5$, it suffices to inspect $d\left(f_{2}\right)$ by symmetry. If $d\left(f_{2}\right)=3$, then $C_{7}$ exists obviously. So assume that $d\left(f_{2}\right) \geqslant 4$, and let $f_{2}=\left[v v_{2} u_{1} \cdots u_{k} v_{3}\right]$ with $k \geqslant 1$ and $k \neq 4$. We first note that $v_{1} \notin\left\{u_{1}, u_{2}, \cdots, u_{k}\right\}$. If $k=3$, we get $C_{7}=v v_{1} v_{2} u_{1} u_{2} u_{3} v_{3} v$. If $k=1$, then $u_{1}$ is identical to at most one of $x, y, v_{0}$, so $C_{7}$ can be always constructed. Assume that $k=2$. If $u_{1}=y$, then $d\left(v_{2}\right)=3$, contradicting the 
definition of $v_{1}$. Thus, suppose that $u_{1} \neq y$. If $u_{2} \neq y$, we get $C_{7}=v v_{1} y v_{2} u_{1} u_{2} v_{3} v$. If $u_{2}=y$, then $u_{1} \notin N\left(v_{1}\right)$, we get $C_{7}=v v_{1} v_{2} u_{1} y x v_{0} v$. Thus, $k \geqslant 5$, that is, $d\left(f_{2}\right) \geqslant 8$.

Claim 8. If $v$ is a 5-vertex adjacent to a bad 4-vertex, then $m_{3}(v) \leqslant 4$; moreover, if $m_{3}(v)=4$, then $m_{8^{+}}(v) \geqslant 1$.

Proof. Assume that $v_{0}, v_{1}, \ldots, v_{4}$ are the neighbors of $v$ in clockwise order, and $v_{1}$ is a bad 4 -vertex. Then $m_{3}\left(v_{1}\right)=4$, implying that both $f_{0}$ and $f_{1}$ are 3 -faces, hence $v_{1} v_{2}, v_{1} v_{0} \in E(G)$. Let $x$ be the neighbors of $v_{1}$ different from $v_{0}, v_{1}, v_{2}$. By symmetry, the proof can be split into two cases below.

Case $1 \quad x \notin\left\{v_{3}, v_{4}\right\}$.

We are going to show that $m_{3}(v) \leqslant 3$ in this case. Assume to the contrary that $m_{3}(v) \geqslant 4$. Without loss of generality, we assume that $d\left(f_{2}\right)=3$ (otherwise, $d\left(f_{4}\right)=3$.) Then at least one of $f_{3}$ and $f_{4}$ is a 3 -face. If $d\left(f_{3}\right)=3$, we get $C_{7}=v v_{0} x v_{1} v_{2} v_{3} v_{4} v$. If $d\left(f_{4}\right)=3$, we get $C_{7}=v v_{4} v_{0} x v_{1} v_{2} v_{3} v$. We always obtain a contradiction.

Case $2 v_{3}=x$.

Clearly, $v_{4} \neq x$ as $G$ is simple. If $d\left(f_{2}\right)=3$, then $d\left(v_{2}\right)=3$, contradicting (P3). Thus, $d\left(f_{2}\right) \geqslant 4$, and therefore it follows that $m_{3}(v) \leqslant 4$. To complete the proof, assume that $m_{3}(v)=4$. This implies that both $f_{3}$ and $f_{4}$ are 3 -faces, hence $v_{0} v_{4}, v_{4} x \in E(G)$. Let $f_{2}=\left[v v_{2} u_{1} \cdots u_{k} x\right]$ with $k \geqslant 1$ and $k \neq 4$. It is easy to see that $v_{0}, v_{1}, v_{4} \notin\left\{u_{1}, u_{2}, \cdots, u_{k}\right\}$ by the planarity of $G$. If $k=1$, we get $C_{7}=v v_{4} v_{0} v_{1} x u_{1} v_{2} v$. If $k=2$, we get $C_{7}=$ $v v_{0} v_{1} x u_{2} u_{1} v_{2} v$. If $k=3$, we get $C_{7}=v v_{1} x u_{3} u_{2} u_{1} v_{2} v$. Thus, $k \geqslant 5$, i.e., $d\left(f_{2}\right) \geqslant 8$.

Claim 9. No two bad 5-vertices are adjacent.

Proof. Assume that $v$ is a bad 5 -vertex with neighbors $v_{0}, v_{1}, \cdots, v_{4}$ in clockwise order which is adjacent to a bad 5-vertex, say $v_{1}$. Let $x$ and $y$ be the other neighbors of $v_{1}$ such that $v, v_{0}, x, y, v_{2}$ are arranged around $v_{1}$ in clockwise order. If at least one of $x$ and $y$ does not belong to $N(v)$, then a 7-cycle is easily established. Otherwise, it is easy to derive that $x=v_{3}$ and $y=v_{4}$, which is impossible by the planarity of $G$.

Using Euler's formula $|V(G)|-|E(G)|+|F(G)|=2$, we have

$$
\sum_{v \in V(G)}(3 d(v)-8)+\sum_{f \in F(G)}(d(f)-8)=-16 .
$$

We define an initial weight function $w$ by $w(v)=3 d(v)-8$ for a vertex $v \in V(G)$, and $w(f)=d(f)-8$ for a face $f \in F(G)$. It follows from equality (1) that the total sum of weights is -16 . Then, we will define appropriate discharging rules and redistribute weights accordingly. Once the discharging is finished, a new weight function $w^{\prime}$ is produced. However the total sum of weights is kept fixed when the discharging is in process. Nevertheless, we can show that $w^{\prime}(x) \geqslant 0$ for all $x \in V(G) \cup F(G)$. This leads to the following obvious contradiction:

$$
0 \leqslant \sum_{x \in V(G) \bigcup F(G)} w^{\prime}(x)=\sum_{x \in V(G) \bigcup F(G)} w(x)=-16,
$$


and hence demonstrates that no such counterexample can exist.

Our discharging rules are defined as follows.

(R1) Every vertex $v$ sends $\frac{5}{3}$ to each incident 3 -face, 1 to each incident 4 -face, $\frac{3}{5}$ to each incident 5 -face, and $\frac{1}{3}$ to each incident 6 -face.

(R2) Every 6-vertex $v$ sends $\frac{7}{3}$ to each adjacent 2-vertex, $\frac{2}{d_{3}(v)+d_{4}(v)+d_{5}(v)}$ to each adjacent 3-, 4- or 5-vertex, with one exception: if $v$ is adjacent to a 3 -vertex $x$ and a 5 -vertex $y$ with $x y \in E(G)$, then $v$ sends 2 to $x$ and $\frac{1}{3}$ to $y$.

(R3) Every master sends 1 to its slave.

Let $\alpha(v)$ denote the resultant weight of a vertex $v$ after (R1)-(R3) are carried out. Then we do the following additional assignments:

(R4) Every 5-vertex $v$ with $\alpha(v)>0$ sends $\frac{\alpha(v)}{d_{3}(v)+d_{4}(v)}$ to each adjacent 3 - or 4 -vertex.

(R5) Every 6-vertex $v$ with $\alpha(v)>0$ sends $\frac{\alpha(v)}{d_{5}(v)}$ to each adjacent bad 5 -vertex.

For $x, y \in V(G) \cup F(G)$, let $\tau(x \rightarrow y)$ denote the amount of weights transferred from $x$ to $y$ according to our discharging rules. A vertex $x$ is called small if $2 \leqslant d(x) \leqslant 5$.

Observation 1 Under (R2), every 6-vertex sends at most $\frac{7}{3}$ to its adjacent small vertices.

Proof. Let $v$ be a 6 -vertex in $G$, and let $s(v)$ denote the sum of weights that $v$ has sent to its small adjacent vertices according to (R2). It suffices to inspect that $s(v) \leqslant \frac{7}{3}$.

If $v$ is adjacent to a 2-vertex $u$, then $d_{6}(v)=5$ by (P5), i.e., $d_{3}(v)+d_{4}(v)+d_{5}(v)=0$. By (R2), $v$ sends $\frac{7}{3}$ to $u$ and nothing to other neighbors. Therefore, $s(v)=\frac{7}{3}$. Otherwise, assume that $d_{2}(v)=0$. If $v$ is adjacent to a 3-vertex $x$ and a 5-vertex $y$ with $x y \in E(G)$, then $v$ cannot be adjacent to other small vertices by (P5). That is, $d_{3}(v)=d_{5}(v)=1$ and $d_{4}(v)=0$. By $(\mathrm{R} 1), s(v)=\tau(v \rightarrow x)+\tau(v \rightarrow y)=2+\frac{1}{3}=\frac{7}{3}$. If $v$ is not adjacent to such vertices $x$ and $y$, then $s(v) \leqslant 2$ by $(\mathrm{R} 2)$.

Observation 2 Let $v$ be a 6 -vertex and $u$ a small vertex adjacent to $v$. Then, after (R2) was carried out, we have the following:

(1) If $d(u)=3$, then $\tau(v \rightarrow u) \geqslant 1$.

(2) If $d(u)=4$, then $\tau(v \rightarrow u) \geqslant \frac{2}{3}$.

(3) If $d(u)=5$ and $v$ is adjacent to a 3-vertex $x$ such that $u x \in E(G)$, then $\tau(v \rightarrow$ $u)=\frac{1}{3}$; otherwise $\tau(v \rightarrow u) \geqslant \frac{1}{2}$.

Proof. Suppose that $v$ is a 6 -vertex adjacent to a vertex $u$ with $3 \leqslant d(u) \leqslant 5$.

(1) Assume that $d(u)=3$. By (P5), we see that $d_{2}(v)=0, d_{6}(v) \geqslant 4$, hence $d_{3}(v)+d_{4}(v)+d_{5}(v) \leqslant 2$. If $v$ is adjacent to a 5 -vertex $y$ such that $u y \in E(G)$, we have $\tau(v \rightarrow u)=2$ by $(\mathrm{R} 2)$. Otherwise, $\tau(v \rightarrow u)=2 /\left(d_{3}(v)+d_{4}(v)+d_{5}(v)\right) \geqslant 1$.

(2) Assume that $d(u)=4$. It is easy to see that $d_{2}(v)=0$ by $(\mathrm{P} 5)$. If $d_{3}(v) \geqslant 1$, then it follows from the proof of $(1)$ that $\tau(v \rightarrow u) \geqslant 1$. Thus, assume that $d_{3}(v)=0$. Since $d_{4}(v) \geqslant 1,(\mathrm{P} 5)$ asserts that $d_{6}(v) \geqslant 3$, which implies that $d_{4}(v)+d_{5}(v) \leqslant 3$. Consequently, $\tau(v \rightarrow u)=2 /\left(d_{4}(v)+d_{5}(v)\right) \geqslant \frac{2}{3}$. 
(3) Assume that $d(u)=5$. Again, (P5) guarantees that $d_{2}(v)=0$. If $v$ is adjacent to a 3-vertex $x$ such that $u x \in E(G)$, then $\tau(v \rightarrow u)=\frac{1}{3}$ by (R2). Otherwise, when $d_{3}(v)+d_{4}(v) \geqslant 1$, the result follows from the proofs of Cases (1) and (2). So assume that $d_{3}(v)=d_{4}(v)=0$. By VAL, $d_{6}(v) \geqslant 2$, so $d_{5}(v) \leqslant 4$. This shows $\tau(v \rightarrow u)=2 / d_{5}(v) \geqslant$ $\frac{1}{2}$.

We carry out (R1)-(R5) in $G$. Let $w^{\prime}$ denote the resultant weight function after discharging was finished. It remains to verify that $w^{\prime}(x) \geqslant 0$ for all $x \in V(G) \cup F(G)$.

Let $f \in F(G)$. Since $G$ is 2-connected, $b(f)$ forms a cycle. This means that $f$ is incident to $d(f)$ distinct vertices. Since $G$ is $C_{7}$-free, $d(f) \neq 7$. If $d(f)=3$, then each of its boundary vertices gives it exactly $\frac{5}{3}$ by (R1). Thus, $w^{\prime}(f)=3-8+3 \times \frac{5}{3}=0$. If $4 \leqslant$ $d(f) \leqslant 6$, we have a similar examination. If $d(f) \geqslant 8$, then $w^{\prime}(f)=w(f)=d(f)-8 \geqslant 0$.

Let $v \in V(G)$. Then $2 \leqslant d(v) \leqslant 6$. Let $v_{0}, v_{1}, \cdots, v_{d(v)-1}$ denote the neighbors of $v$ in clockwise order. For $0 \leqslant i \leqslant d(v)-1$, we use $f_{i}$ to represent the incident face of $v$ with $v v_{i}$ and $v v_{i+1}$ as boundary edges, where indices are taken modulo $d(f)$. The proof is divided into the following five cases.

(1) $d(v)=2$.

It is easy to see that $d_{6}(v)=2$ by $(\mathrm{P} 5), m_{3}(v) \leqslant 1$ since $G$ is simple. By (R1) and (R2), we get that $w^{\prime}(v) \geqslant(3 \times 2-8)+2 \times \frac{7}{3}-\frac{5}{3}-1=0$.

(2) $d(v)=3$.

Then $w(v)=1$. By $(\mathrm{P} 2), d_{4^{-}}(v)=0$, hence $d_{5}(v)+d_{6}(v)=3$. Without loss of generality, assume that $d\left(v_{1}\right) \leqslant d\left(v_{2}\right) \leqslant d\left(v_{0}\right)$. By VAL, $d\left(v_{1}\right) \geqslant 5$ and $d\left(v_{2}\right)=d\left(v_{0}\right)=6$.

If $d\left(v_{1}\right)=6$, then each $v_{i}$, for $i=0,1,2$, is adjacent to at most two $5^{-}$-vertices by VAL, and at least one $v_{i}$ is adjacent to only one $5^{-}$-vertex by Lemma 4 . Thus, by (R1), (R2) and Observation 2(1), $w^{\prime}(v) \geqslant 1+2+2 \times 1-3 \times \frac{5}{3}=0$.

Assume that $d\left(v_{1}\right)=5$. Then $d_{6}\left(v_{1}\right)=4$. If $m_{3}(v)=0$, then $w^{\prime}(v) \geqslant 1+2 \times 1-3=0$ by (R1) and Observation 2(1).

- Assume that $m_{3}(v)=1$. If $f_{1}$ is a 3 -face, then $\tau\left(v_{2} \rightarrow v\right)=2$ by (R2). If $f_{0}$ is a 3 -face, then $\tau\left(v_{0} \rightarrow v\right)=2$ by (R2). It turns out that $w^{\prime}(v) \geqslant 1+2+1-\frac{5}{3}-2 \times 1=\frac{1}{3}$ by (R1) and Observation $2(1)$. If $f_{2}$ is a 3 -face, then $m_{3}\left(v_{1}\right) \leqslant 3$. After (R1)-(R3), $\alpha\left(v_{1}\right) \geqslant(3 \times 5-8)+4 \times \frac{1}{2}-3 \times \frac{5}{3}-2 \times 1=2$ by Observation $2(3)$. By $(\mathrm{R} 4), \tau\left(v_{1} \rightarrow v\right) \geqslant 2$. Hence $w^{\prime}(v) \geqslant 1+2 \times 1+2-\frac{5}{3}-2 \times 1=\frac{1}{3}$.

- Assume that $m_{3}(v) \geqslant 2$. If both $f_{0}$ and $f_{1}$ are 3 -faces, then $\tau\left(v_{i} \rightarrow v\right)=2$ for $i=0,2$ by $(\mathrm{R} 2)$, hence $w^{\prime}(v) \geqslant 1+2 \times 2-3 \times \frac{5}{3}=0$. Otherwise, we may suppose that $d\left(f_{1}\right)=d\left(f_{2}\right)=3$ and $d\left(f_{0}\right) \geqslant 4$ by symmetry. By $(\mathrm{R} 2), \tau\left(v_{2} \rightarrow v\right)=2$. Since $m_{3}\left(v_{1}\right) \leqslant 4$, after (R1)-(R3), we obtain that $\alpha\left(v_{1}\right) \geqslant 7-4 \times \frac{5}{3}-1+3 \times \frac{1}{2}=\frac{5}{6}$ by Observation $2(3)$. By $(\mathrm{R} 4), \tau\left(v_{1} \rightarrow v\right) \geqslant \frac{5}{6}$. Therefore, $w^{\prime}(v) \geqslant 1+2+\frac{5}{6}+1-2 \times \frac{5}{3}-1=\frac{1}{2}$.

(3) $d(v)=4$.

Then $w(v)=4$, and $d_{2}(v)=d_{3}(v)=0$ by (P3). By VAL, $d_{6}(v) \geqslant 2$. If $m_{3}(v) \leqslant 2$, then $w^{\prime}(v) \geqslant 4+2 \times \frac{2}{3}-2 \times \frac{5}{3}-2 \times 1=0$ by (R1) and Observation 2(2).

- Assume that $m_{3}(v)=3$, say $d\left(f_{i}\right)=3$ for $i=1,2,3$, and $d\left(f_{0}\right) \geqslant 4$. If $d_{6}(v) \geqslant 3$, then $w^{\prime}(v) \geqslant 4+3 \times \frac{2}{3}-3 \times \frac{5}{3}-1=0$. So suppose that $d_{6}(v)=2$ by VAL, which implies that $d_{5}(v)=2$. By symmetry, we need to consider the following four possibilities:

If $d\left(v_{1}\right)=d\left(v_{2}\right)=5$, then $d_{6}\left(v_{1}\right)=3, d_{5}\left(v_{1}\right)=d_{4}\left(v_{1}\right)=1$, and $d_{3}\left(v_{1}\right)=0$. After 
(R1)-(R3), $\alpha\left(v_{1}\right) \geqslant 7-4 \times \frac{5}{3}-1+3 \times \frac{1}{2}=\frac{5}{6}$. By (R4), $\tau\left(v_{1} \rightarrow v\right) \geqslant \frac{5}{6}$ and hence $w^{\prime}(v) \geqslant 4+2 \times \frac{2}{3}+\frac{5}{6}-3 \times \frac{5}{3}-1=\frac{1}{6}$ by (R1) and Observation 2(2).

If $d\left(v_{0}\right)=d\left(v_{1}\right)=5$, then $d_{6}\left(v_{i}\right) \geqslant 3, d_{3}\left(v_{i}\right)=0$, and $d_{4}\left(v_{i}\right) \leqslant 2$ for $i=0,1$. After (R1)-(R3), $\alpha\left(v_{i}\right) \geqslant 7-4 \times \frac{5}{3}-1+3 \times \frac{1}{2}=\frac{5}{6}$. By (R4), $\tau\left(v_{i} \rightarrow v\right) \geqslant \frac{5}{12}$ for $i=0,1$. Hence $w^{\prime}(v) \geqslant 4+2 \times \frac{2}{3}+2 \times \frac{5}{12}-3 \times \frac{5}{3}-1=\frac{1}{6}$.

If $d\left(v_{1}\right)=d\left(v_{3}\right)=5$, then for $i \in\{1,3\}, d_{6}\left(v_{i}\right) \geqslant 3, d_{4}\left(v_{i}\right) \leqslant 2, d_{3}\left(v_{i}\right)=0$. Note that $v_{1}$ is adjacent to a 6 -vertex which is adjacent to the 4 -vertex $v$, and $v_{3}$ is adjacent to two 6 -vertices which are adjacent to the 4 -vertex $v$. After (R1)-(R3), $\alpha\left(v_{1}\right) \geqslant 7-4 \times \frac{5}{3}-1+$ $2 \times \frac{1}{2}+\frac{2}{3}=1$, and $\alpha\left(v_{3}\right) \geqslant 7-5 \times \frac{5}{3}+\frac{1}{2}+2 \times \frac{2}{3}=\frac{1}{2}$ by Observation 2 and its proof. By $(\mathrm{R} 4), \tau\left(v_{1} \rightarrow v\right) \geqslant \frac{1}{2}$, and $\tau\left(v_{3} \rightarrow v\right) \geqslant \frac{1}{4}$, hence $w^{\prime}(v) \geqslant 4+2 \times \frac{2}{3}+\frac{1}{2}+\frac{1}{4}-3 \times \frac{5}{3}-1=\frac{1}{12}$ by $(\mathrm{R} 1)$ and Observation $2(2)$.

If $d\left(v_{2}\right)=d\left(v_{3}\right)=5$, then for $i \in\{2,3\}, d_{6}\left(v_{i}\right)=3, d_{4}\left(v_{i}\right)=d_{5}\left(v_{i}\right)=1, d_{3}\left(v_{i}\right)=0$, and $v_{i}$ is adjacent to a 6 -vertex which is adjacent to the 4 -vertex $v$. After (R1)-(R3), $\alpha\left(v_{i}\right) \geqslant 7-5 \times \frac{5}{3}+2 \times \frac{1}{2}+\frac{2}{3}=\frac{1}{3}$ by Observation 2 and its proof. By (R4), $\tau\left(v_{i} \rightarrow v\right) \geqslant \frac{1}{3}$ for $i=2,3$, hence $w^{\prime}(v) \geqslant 4+2 \times \frac{2}{3}+2 \times \frac{1}{3}-3 \times \frac{5}{3}-1=0$ by (R1) and Observation 2(2).

- Assume that $m_{3}(v)=4$. Then $v$ is bad. If $d_{6}(v)=4$, then $w^{\prime}(v) \geqslant 4+4 \times \frac{2}{3}-4 \times \frac{5}{3}=0$ by (R1) and Observation 2(2). If $v$ is adjacent to a 4 -vertex, say $v_{1}$, then by Lemma 3 , every vertex in $N\left(N\left(v, v_{1}\right)\right) \backslash\left\{v, v_{1}\right\}$ is of degree 6 . It follows that $\tau\left(v_{3} \rightarrow v\right)=2$ and $\tau\left(v_{i} \rightarrow v\right)=1$ for $i=0,2$, hence $w^{\prime}(v) \geqslant 4+2+2 \times 1-4 \times \frac{5}{3}=\frac{4}{3}$. So assume that $d_{4}(v)=0,2 \leqslant d_{6}(v) \leqslant 3$ by VAL, hence $1 \leqslant d_{5}(v) \leqslant 2$. Let $d\left(v_{1}\right)=5$. Then $d_{6}\left(v_{1}\right) \geqslant 3$. By Claim 8, $y$ sends at most $7\left(=\max \left\{3 \times \frac{5}{3}+2,4 \times \frac{5}{3}\right\}\right)$ to incident faces. We see that $\alpha\left(v_{1}\right) \geqslant 7-7+3 \times \frac{1}{2}=\frac{3}{2}$ by Observation $2(3)$. By $(\mathrm{R} 4), \tau\left(v_{1} \rightarrow v\right) \geqslant \frac{3}{4}$. If $d_{5}(v)=1$, then $d_{6}(v)=3$, and therefore $w^{\prime}(v) \geqslant 4+3 \times \frac{2}{3}+\frac{3}{4}-4 \times \frac{5}{3}=\frac{1}{12}$.

Now, assume that $d_{5}(v)=2$. We have two subcases to be handled. If $d\left(v_{2}\right)=5$, then $d_{6}\left(v_{i}\right)=3, d_{4}\left(v_{i}\right)=1$ and $d_{3}\left(v_{i}\right)=0$ for $i=1,2$. After (R1)-(R3), $\alpha\left(v_{i}\right) \geqslant 7-7+3 \times \frac{1}{2}=\frac{3}{2}$. By $(\mathrm{R} 4), \tau\left(v_{i} \rightarrow v\right) \geqslant \frac{3}{2}$ for $i=1,2$. Hence $w^{\prime}(v) \geqslant 4+2 \times \frac{2}{3}+2 \times \frac{3}{2}-4 \times \frac{5}{3}=\frac{5}{3}$. If $d\left(v_{3}\right)=5$, then $d_{6}\left(v_{i}\right) \geqslant 3, d_{4}\left(v_{i}\right) \leqslant 2, d_{3}\left(v_{i}\right)=0$ for $i=1,3$. Thus, $\alpha\left(v_{i}\right) \geqslant 7-7+3 \times \frac{1}{2}=\frac{3}{2}$, $\tau\left(v_{i} \rightarrow v\right) \geqslant \frac{3}{4}$ for $i=1,3$, and $w^{\prime}(v) \geqslant 4+2 \times \frac{2}{3}+2 \times \frac{3}{4}-4 \times \frac{5}{3}=\frac{1}{6}$ accordingly.

(4) $d(v)=5$.

Then $w(v)=7$. We note that $d_{6}(v) \geqslant 2, d_{2}(v)=0$, and $d_{3}(v) \leqslant 1$ by VAL. If $m_{3}(v) \leqslant 4$, then $\alpha(v) \geqslant 7+2 \times \frac{1}{3}-4 \times \frac{5}{3}-1=0$ by (R1) and Observation 2(3). So assume that $m_{3}(v)=5$. If $d_{3}(v)=1$, then $d_{6}(v)=4$ and henceforth $\alpha(v) \geqslant 7+4 \times \frac{1}{3}-5 \times \frac{5}{3}=0$. Furthermore, assume that $d_{3}(v)=0$. If $d_{6}(v) \geqslant 3$, then $\alpha(v) \geqslant 7+3 \times \frac{1}{2}-5 \times \frac{5}{3}=\frac{1}{6}$ by Observation 2(3). Otherwise, we conclude that $d_{6}(v)=2$ and $d_{5}(v)=3$. This shows that $v$ is a bad 5-vertex. Let $y$ be an arbitrary 6-vertex adjacent to $v$. By Claim $7, m_{8^{+}}(y) \geqslant 2$. Thus, $\alpha(y) \geqslant(3 \times 6-8)-4 \times \frac{5}{3}-\frac{7}{3}=1$ by Observation 1 . Note that $y$ is adjacent to at most three bad 5 -vertices by Claim 9 . Thus, $\tau(y \rightarrow v) \geqslant \frac{1}{3}$ by $(\mathrm{R} 5)$, and hence $w^{\prime}(v) \geqslant 7+2 \times \frac{1}{2}+2 \times \frac{1}{3}-5 \times \frac{5}{3}=\frac{1}{3}$.

(5) $d(v)=6$.

We see that $w(v)=10, d_{6}(v) \geqslant 2, d_{2}(v) \leqslant 1$, and $m_{3}(v) \leqslant 4$ since $G$ contains no 7 -cycles. Instead of showing that $w^{\prime}(v) \geqslant 0$, we need only to prove that $\alpha(v) \geqslant 0$. If $m_{3}(v) \leqslant 1$, then $\alpha(v) \geqslant 10-\frac{7}{3}-1 \times \frac{5}{3}-5 \times 1=1$ by (R1) and Observation 1 . If $m_{3}(v)=2$ and $v$ is not the master of any vertex, then $\alpha(v) \geqslant 10-\frac{7}{3}-2 \times \frac{5}{3}-4 \times 1=\frac{1}{3}$. 
If $m_{3}(v)=2$, and moreover $v$ is the master of some vertex, then by the analysis following the proof of Claim 5, we know that $m_{4}(v)=2$ and $v$ has exactly one slave. Thus, $\alpha(v) \geqslant 10-\frac{7}{3}-2 \times \frac{5}{3}-2 \times 1-2 \times \frac{3}{5}-1=\frac{2}{15}$ by (R1) and (R3). If $m_{3}(v)=3$, then by Claim $6, m_{4}(v) \leqslant 2$, or $m_{4}(v)=3$ and $d_{2}(v)=d_{3}(v)=0$. For the former, $\alpha(v) \geqslant 10-\frac{7}{3}-3 \times \frac{5}{3}-2 \times 1-\frac{3}{5}=\frac{1}{15}$. For the latter, $v$ sends at most 2 to adjacent small vertices, thus $\alpha(v) \geqslant 10-2-3 \times \frac{5}{3}-3 \times 1=0$.

Finally, assume that $m_{3}(v)=4$. If $m_{4}(v)=2$, then $v$ is a slave of some vertex, hence $\alpha(v) \geqslant 10+1-\frac{7}{3}-4 \times \frac{5}{3}-2 \times 1=0$ by (R3) and Observation 1 . If $m_{4}(v)=1$, then by Claim $5(2), m_{8^{+}}(v)=1$ and hence $\alpha(v) \geqslant 10-\frac{7}{3}-4 \times \frac{5}{3}-1=0$. If $m_{4}(v)=0$, then by Claim $5(3)$ and $(4), m_{5}(v)=1$ and $m_{6^{+}}(v)=1$, or $m_{6^{+}}(v)=2$. Thus, we always have $\alpha(v) \geqslant 10-\frac{7}{3}-4 \times \frac{5}{3}-\frac{3}{5}-\frac{1}{3}=\frac{1}{15}$.

\section{References}

[1] Y. Bu and W. Wang. Some sufficient conditions for a planar graph of maximum degree six to be class one. Discrete Math., 306: 1440-1445, 2006.

[2] X. Li, R. Luo, and J. Niu. A note on class one graphs with maximum degree six. Discrete Math., 306: 1450-1455, 2006.

[3] R. Luo and C. Q. Zhang. Edge coloring of graphs with small average degree. Discrete Math., 275: 207-218, 2004.

[4] D. Sanders and Y. Zhao. Planar graphs of maximum degree seven are class I. J. Combin. Theory Ser. B, 83: 201-212, 2001.

[5] D. Sanders and Y. Zhao. Coloring edges of graphs embedded in a surface of characteristic zero. J. Combin. Theory Ser. B, 87: 254-263, 2003.

[6] V. G. Vizing. Critical graphs with given chromatic class. Metody Diskret. Analiz., 5: 9-17, 1965 (in Russian).

[7] W. Wang and Y. Chen. A sufficient for a planar graph to be class 1. Theoret. Comput. Sci., 385: 71-77, 2007.

[8] Y. Wang, Y. Chen, and W. Wang. A new sufficient condition for a planar graph of maximum degree six to be Class 1. Sci. Sin. Math., 40: 1129-1136, 2010 (In Chinese).

[9] L. Zhang. Every planar graph with maximum degree 7 is of class one. Graphs Combin., 16: 467-495, 2000.

[10] G. Zhou. A note on graphs of class I. Discrete Math., 263: 339-345, 2003. 